\title{
UNA CARTOGRAFÍA SOCIAL DEL GÉNERO EN EDUCACIÓN
}

\author{
Nelly P. StromQuist
}

\begin{abstract}
RESUMEN: Comprender cómo se trata el género en los sistemas educativos requiere una visión holística de los actores y espacios involucrados. La cartografía social ayuda a visualizar los múltiples terrenos y puntos de encuentro y desencuentro. El artículo resalta tanto la naturaleza independiente de los espacios privados y públicos como la interacción entre ellos. El espacio privado reduce las posibilidades de ciudadanía de la mujer. El espacio público, dominado por el Estado y las agencias internacionales, promueve la paridad de acceso a la escuela y es reacio a considerar curriculos contestarios, como la educación sexual y los derechos económicos y sociales de las mujeres. Los actores públicos no estatales apoyan la educación de las mujeres adultas pero despliegan esfuerzos débiles hacia la educación formal. Este contexto físico, aunado a tiempos de modernización y globalización que fomentan prácticas competitivas, anticipa un futuro incierto en la redefinición del género en las relaciones sociales.
\end{abstract}

Palabras clave: Género. Educación. Cartografia social. Espacio público. Espacio privado.

\section{A SOCIAL MAPPING OF GENDER IN EDUCATION}

ABSTRACT: Understanding how the concept of gender is dealt with in educational systems requires a holistic comprehension of its actors and spaces. Social mapping helps visualize the many grounds and points of convergence and divergence. This paper highlights both the independent natures of private and public spaces, and the interaction between them. The private sphere (the home) definitely reduces women's possibilities for full citizenship. Dominated by the state and international agencies, the public space promotes a partial remedy - parity in access to schooling - but ignores curricula that

* Ph.D. em Educação pela Stanford University, Rossier School of Education e University of Southern California (EUA).E-mail: nellystromquist@juno.com 
Una cartografía social del género en educación

question the status quo, such as sex education and the economic and social rights of women. The non-state public actors support the education of adult women but only deploy weak efforts to modify formal school-age education. This physical context, combined with current times of modernization and globalization that foster competitive practices, forecasts an uncertain future in the redefinition of the social relations of gender.

Key words: Gender. Education. Social mapping. Public sphere. Private sphere.

\section{Introducción}

E

n esta presentación ofrezco una visión holística de género en educación, repasando cómo es contextualizado este fenómeno y cómo funciona en los hechos. Examinaré sus perfiles globales y sus implicancias en políticas públicas, utilizando el marco conceptual de cartografía social. Originalmente desarrollado en la disciplina de geografía, este marco permite al investigador considerar: territorio, ubicación, actores institucionales e individuales que ocupan el espacio social, la manera en que estos actores asumen problemáticas y soluciones; y la interrelación entre todos éstos (Siekierska, c1995). ${ }^{1}$ Aplicada al análisis del género desde una perspectiva política, la cartografía social nos ayuda a delinear los diversos terrenos e instituciones, su relativo tamaño, su intersección e influencia. Propongo dos tesis: (1) el género requiere un mapeo inclusivo a fin de entender avances y retrocesos del cambio social hacia nuevas relaciones sociales; y (2) la interconexión entre espacios sociales e institucionales es esencial para el progreso de género. Para ello uso fuentes internacionales pero me centro en la experiencia latinoamericana.

\section{Teorías sobre el género}

La teoría de género originalmente apuntaba hacia las asimetrías de poder entre mujeres y hombres; a través del tiempo su complejidad se ha incrementado y se ha visto fracturada por diferencias en sus premisas básicas. Existen diferencias en la percepción de la naturaleza de la opresión y estos grados de subordinación se originan en condiciones ideológicas y materiales, unidas al patriarcado en la construcción de categorías binarias de mujer y hombre y a la afirmación de dis- 
curso y representación, como los principales procesos a través de los cuales la opresión es practicada. Esta comprensión matizada de lo que es la opresión y de cómo se manifiesta ha llevado, en cada caso, a una gama de propuestas para erradicarla. Tensiones significativas pueden ser detectadas entre el principio de igualdad y derecho a ser diferente; entre derechos individuales y colectivos; entre responsabilidades públicas y respeto a la privacidad e intimidad; y entre una perspectiva enraizada en derechos universales y una perspectiva inmersa en sistemas de relaciones sociales (Dore, 1997; Redclift, 1997).

Todas las teorías de género desenmascaran la existencia de la indiscutible opresión de la mujer. Prevalece el entendimiento que considera que el sistema de género está construido en tres niveles: Estructural - sustentado por la división social del trabajo; institucional conformado por normas y regulaciones que guían la distribución de recursos y oportunidades entre hombres y mujeres; y simbólica enmarcada por conceptos, mentalidades y representaciones colectivas de feminidad y masculinidad (Stromquist, 1995; Guzmán, 2003). El concepto de patriarcado no es usado en varias teorías feministas. Sin embargo, este concepto continúa siendo útil para permitirnos capturar una ideología de género discriminante que coloca al hombre como el referente clave. El patriarcado se refiere no sólo al poder que el hombre recibe de su rol adscrito como cabeza de familia, sino también a los privilegios que el Estado le otorga en múltiples áreas de la vida social y política.

Una vez que uno reconoce género como una construcción social, se nos abre la posibilidad de conceptualizar nuestro mundo y crear comportamientos alternativos. Esta ampliación de horizontes sociales - que se refiere fundamentalmente al derecho de ser - también ha llevado a una comprensión extensa de lo que modificar mentalidades de género particularmente exigiría: no solo la modificación de nociones de masculinidad y feminidad, sino también la ratificación de nociones amplias de ciudadanía, incluyendo el acceso al bienestar social y la aceptación de nuevas formas de expresión de la sexualidad de cada persona y de las orientaciones sexuales. El reclamo de las sexualidades estigmatizadas ha desestabilizado la conexión entre cuerpo e identidad (Redclift, 1997), a pesar que la ubicación de homosexualidad al interior de la agenda política del movimiento de mujeres en varios países latinoamericanos, es todavía un tema no resuelto. 
Una cartografía social del género en educación

$\mathrm{Al}$ margen de las diferencias en la conceptualización de género, es claro que género no es solamente un indicador social entre muchos otros; sino que funciona como un principio articulador que afecta profundamente otras interrelaciones sociales. Las desigualdades de género se manifiestan a niveles macro y micro de la cotidianeidad y afecta a los individuos de todas las clases sociales, de manera transversal. Clase social y etnia están intrincadamente entrelazadas con género; pero el género opera con su autonomía propia.

\section{El género en la educación}

Las teorías de género y educación afirman que la escuela está activamente involucrada en la construcción del género. Ellas también reconocen la naturaleza reproductora de la escolaridad, cuyo conocimiento y práctica diaria construye a partir normas tradicionales y valores que colocan masculinidad y feminidad radicalmente en oposición (Dillabough y Arnot, 2000; Lee, 2000; Davies, 2000). Sin embargo, las teorías de género y educación también consideran que el conocimiento puede ser transformador, a través de diálogo constante y cultivo de la concientización.

La educación siempre abre la posibilidad de cambio. La educación expone a los individuos a ideas cosmopolitas, a dominio de la evidencia y a consideraciones éticas. Las ideas no pueden ser completamente controladas. Entrenar a la gente a pensar analíticamente siempre contiene un potencial revolucionario. Pero son necesarios contenido de soporte y contexto para hacer florecer estas ideas. Creciente investigación en colegios los identifica como lugares claves para la creación de masculinidad (Connell 1995 y 2000). La evidencia de países industrializados - los mismos que pueden ser considerados como las sociedades más avanzadas democráticamente en términos de género - señala que la construcción de género en colegios de primaria y secundaria permanece polarizada; pues los niños continúan dominando el espacio del aula y la atención de los profesores; mientras las contribuciones de las niñas son todavía descontadas. El hostigamiento sexual permanece como un problema y existe una persistente gama para la elección de áreas de estudio entre mujeres y hombres (Francis y Skelton, 2001; Dillabough, 20 00).

La escuela es incuestionablemente difícil de cambiar; y tiende a reproducir la masculinidad y feminidad mediante la creación de grupos pares, de igual edad e igual sexo; que pasan significativa cantidad de tiempo juntos y que se perciben los unos a los otros como principales 
referentes individuales y grupales. No es sorprendente que muchos de aquellos que buscan educación con posibilidades emancipadoras dedican su atención a la educación de mujeres adultas mediante la educación no formal y el aprendizaje informal.

\section{El espacio}

El género encuentra espacio de maneras únicas: tanto hombres como mujeres habitan espacios públicos y privados, pero esos espacios les producen impactos radicalmente diferentes. Para la mujer, el primer tipo de espacio privado es su cuerpo y el segundo es el lugar doméstico del hogar. Los espacios públicos son aquellos creados por los actores establecidos y no establecidos dentro de la amplia sociedad (Harcourt, 2001). El gráfico 1 intenta proporcionar un mapeo de tales espacios, al analizar género en educación.

\section{Gráfico 1}

(Espacio público y educación)

\section{Espacio Privado}

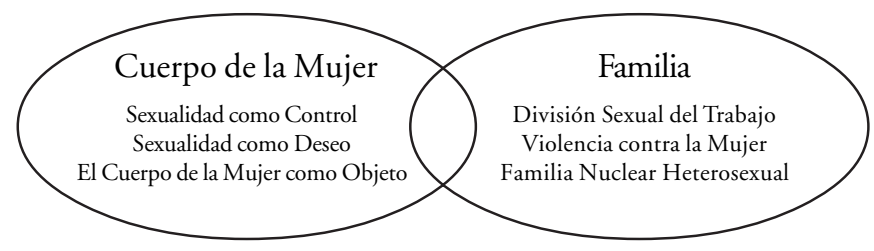

\section{Espacio Público y Educación}

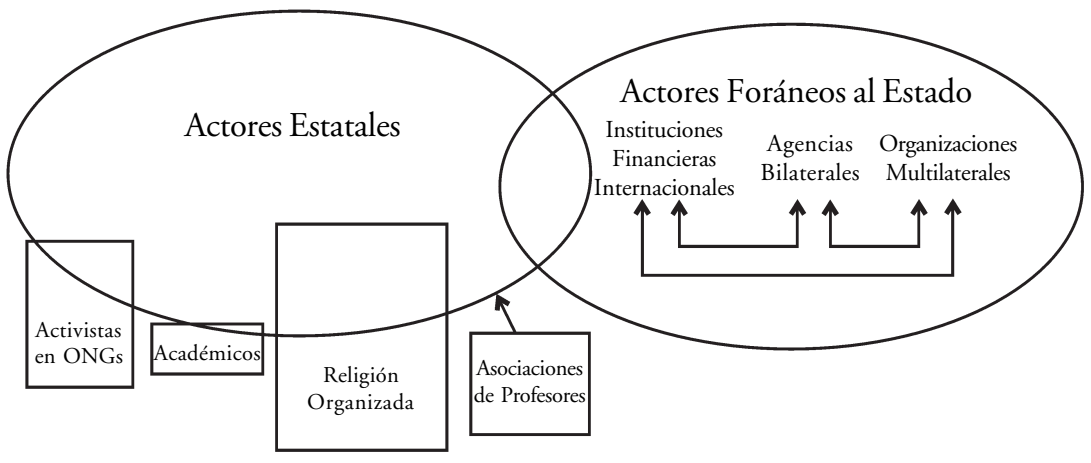


Una cartografía social del género en educación

El espacio privado

\section{Los cuerpos de las mujeres}

El cuerpo es un espacio crítico en el que las mujeres funcionan tanto como seres humanos reproductivos como sujetos y objetos de deseos sexuales. A menudo la sexualidad de las mujeres es reprimida y enmarcada principalmente en su dimensión reproductiva y maternal. Es también utilizada y tratada como mercancía. Las mujeres se encuentran aún luchando para tener autonomía sobre sus propios cuerpos: su derecho a abortar es prohibido e impugnado en muchos países; su derecho a usar contraconceptivos es desafiado por varios grupos religiosos fundamentalistas; $y$ su libertad de movimiento y derecho a elegir un esposo, son todavía negados en muchos países.

Tal control forma la sexualidad de las mujeres como sumisa y receptiva, mientras que la sexualidad de los hombres es naturalizada como agresiva e instintiva (y por lo tanto incontrolable); este doble estándar en la conceptualización de la sexualidad afecta profundamente las oportunidades en la vida de las mujeres porque se extiende a otras formas de comportamiento social. En años recientes se ha progresado en varias naciones al establecer mayor legislación definitiva contra la violación y violencia marital; en un puñado de países, la legislación contra la violación de la pareja se ha promulgado. Sin embargo, en la mayoría de los países se continúa controlando la sexualidad en una variedad de formas: interviniendo en fomentar el control de la fertilidad a través de políticas de planificación familiar basadas en cuotas y blancos demográficos; relegando los temas del deseo sexual de las mujeres y de derechos sexuales a la categoría de tabús sociales; enmarcando la homosexualidad como una abominación o una forma inferior de sexualidad; $y$, especialmente, proscribiendo la mayoría de las formas de aborto. Fine (1988) anota, en el contexto de los Estados Unidos, que ha habido un discurso históricamente silenciado sobre sexualidad, medidas de control de la natalidad y abortos en escuelas públicas; ha habido también una ausencia de discurso sobre el deseo, ya que se han proporcionado en este país tan incompletas formas de educación sexual. Aún más duras observaciones podrían hacerse de muchos otros países.

Los cuerpos de las mujeres son espacios que proveen limitado poder para la protección y defensa de las mujeres mismas. Los cuerpos de las mujeres no son sólo espacios contenciosos, sino espacios que el Esta- 
Nelly P. Stromquist

do y particularmente las políticas educativas explícitamente evitan. La violencia contra las mujeres y la negación de sus derechos - incluyendo la prerrogativa de eludir embarazos no deseados - son parte de las agendas políticas de las mujeres en todo el mundo; aunque, en tiempos recientes, los derechos sexuales y reproductivos se encuentran bajo un ataque frontal por parte de fuerzas conservadoras en el Oeste. Una dificultad clave del manejo del cuerpo es que las mujeres han sido socializadas para reservar este espacio para sus interrelaciones más íntimas. Por lo tanto, hablar sobre sexualidad es visto como inapropiado.

\section{La familia}

Lo doméstico es cultural y económicamente específico, pero las mujeres tienden a ser mucho más responsables que los hombres en cuidar a la familia (Ashfar, 1987). En el hogar, las construcciones sociales de la feminidad colocan a las mujeres como madres a tiempo completo, administradoras de la familia y básicamente esposas. Hay una creciente conciencia de que las normas de la vida doméstica refuerzan patrones de sumisión dentro de la familia; y que estas normas se extienden a las instituciones fuera del hogar. Fue una clave de discernimiento intelectual del movimiento de mujeres en los años 60s, darse cuenta que "lo personal es lo político". ${ }^{2}$ Actualmente se entiende que las demandas de espacio y tiempo requeridos por la familia resultan en prácticas que afectan la capacidad de la mujer para introducirse y actuar en el mundo político. Así, de hecho, el mundo público y el mundo privado se encuentran interconectados. Claramente, la política no existe solamente fuera del hogar; deriva de transacciones que se originan a niveles micro, incluyendo aquellas dentro del hogar (Pateman, 1988; Bowser y Patton, 2004).

La construcción social de género, sin embargo, implica que la familia puede ser utilizada tanto para reproducir como para transformar normas de género dominantes. La investigación muestra que las familias son también espacios de resistencia y negociación, porque las diferencias de privilegio y poder pueden ser cuestionadas. A través de las acciones del movimiento de mujeres, actualmente existe más atención por parte del gobierno, así como legislación concomitante para acabar con la violencia doméstica, especialmente, la violencia contra las mujeres; desafortunadamente, "pocas medidas inciden en las raíces de las causas de la violencia o desafían los intereses creados de las normas culturales que 
Una cartografía social del género en educación

permiten visualizar la violación y la violencia doméstica como asuntos de familia privados" (Grossman et al, 2005, p. 10). La implementación tarda tras las pocas leyes que mejoran el espacio doméstico.

La familia permanece como un espacio utilizado por el Estado para promover una visión particular de las mujeres y una definición específica de familia. El sistema educativo, a su vez, invoca el espacio de familia principalmente para sostener una versión de la familia - la familia nuclear y heterosexual - y para enlazar a las mujeres con la maternidad y las funciones del cuidado. Para las mujeres, el reto es cómo transformar temas que están ahora en el dominio privado de costumbres y creencias en temas que deberían ser resueltos en el espacio público y así ser tratadas mediante reglas claras y justas.

\section{El espacio público}

El espacio público incluye el Estado y la sociedad civil. El Estado ocupa el espacio público mediante actores tanto de Estados nacionales como Estados foráneos. Estos ultimos funcionan mediante las instituciones financieras internacionales; las agencias de desarrollo bilateral; y las agencias multilaterales; estas agencias no son instituciones autónomas y su comportamiento está "condicionado y delimitado por las decisiones y el poder del Estado" de sus respectivos Estados miembros (Krasner, 1985 , p. 28). La sociedad civil ocupa el espacio público como grupos organizados no gubernamentales, tales como grupos de defensa de las mujeres, de religiones institucionalizadas, $\mathrm{y}-$ en el contexto de temas educativos - los sindicatos docentes.

\section{Actores estatales}

Todas las conferencias mundiales de las Naciones Unidas desde 1990 invocan la educación como el mecanismo clave para obtener igualdad y crecimiento económico. Adicionalmente, importantes convenciones sobre los derechos políticos de las mujeres se han firmado y ratificado. A pesar de que el Estado es reacio a expandir apropiadamente los derechos de las mujeres, algunas victorias notables se han logrado. La Convención para Eliminar Todas las Formas de Discriminación contra las Mujeres (CEDAW, por sus siglas en inglés) ya había sido firmada por 179 de los 191 países de las Naciones Unidas en 2004. Sin embargo, repre- 
Nelly P. Stromquist

senta la convención con el mayor número de objeciones, pues 50 países firmantes no aceptan el documento en su totalidad. ${ }^{3}$

Las políticas para el cambio social en género tienden a darse sin investigaciones de base $y$, juzgando a partir de sus persistentes deficientes implementaciones, mayormente con intenciones simbólicas; esto es, a fin de ganar terreno para legitimar al gobierno. Hoy en día se concede mucha importancia al concepto de gobierno democrático: necesidad de la gente a acceder a los derechos humanos, a preocuparse por las decisiones que afectan sus vidas, a asegurarse que sus decidores sean fiables y a tener acceso a normas, instituciones y prácticas inclusivas y justas. También se considera generalmente que debería existir equidad entre las mujeres y los hombres en las esferas privadas y públicas de la vida (UNDP, 2002). A pesar de estas señales progresistas, las discusiones resultantes sobre democracia no consideran ni género, ni la naturaleza de género propia de la democracia contemporánea; en particular, la manera en que el mundo privado se entrelaza con el mundo público y de esa manera, con la ciudadanía. Más aún, con la emergencia de un único importante poder en años recientes, ha habido un retroceso en la conceptualización y respaldo de los derechos de las mujeres. Notoriamente, en este sentido, es la reciente posición de los Estados Unidos en el encuentro en Beijing+10 (2005), al evaluar el progreso en la Plataforma de Acción (un documento que propone áreas específicas para la acción gubernamental, incluyendo educación). Los Estados Unidos buscaron introducir una enmienda al afirmar que los objetivos de la Plataforma de Acción "no crean ningún derecho humano internacional nuevo y que ellos no incluyen el derecho al aborto". Se desestimó la enmienda por falta de apoyo de otros países, especialmente aquellos de la Unión Europea.

En el área de educación, los gobiernos nacionales y las agencias internacionales son actores de estado cruciales. Las políticas educativas estatales se limitan a igualdad de oportunidades, enmarcando igualdad de oportunidades como acceso a la educación y, particularmente, acceso a educación primaria. Considerando que la igualdad de oportunidades se concentra en que las mujeres a accedan a la escuela y completen su educación, estas políticas reducen género a sexo. Y dado que en varios países, particularmente en América Latina, el ingreso de las jóvenes sobrepasa al de los jóvenes (en márgenes pequeños) en el ámbito de escuela secundaria y universidad, los gobiernos de esa región asumen la posición que género no es un problema educativo en sus países. 
Una cartografía social del género en educación

A pesar que los Estados proponen incrementar el acceso a la educación de las niñas, existe débil evidencia de que medidas nacionales específicas se hayan utilizado para acelerar su acceso a la escuela. Las mujeres han ido ganando mayor acceso a la educación en comparación con el pasado; esto parece ocurrir mayormente como resultado del cambio de las condiciones sociales y de la expansión general de la educación formal, que acerca la escuela a la comunidad. Debe tomarse en cuenta que, en lo que corresponde al Estado, existe también tendencia a considerar indistinto igualdad y equidad. En lugar de promover igualdad, muchos gobiernos proponen equidad, confundiendo de esta manera los medios con los fines. La equidad debe hacer referencia a los medios por los cuales se obtiene la igualdad. Así, en lugar de un planteamiento indiferenciado, la equidad propone tratamiento diferenciado. La equidad real, como medidas compensatorias para lograr cambios en las condiciones educativas de las niñas, ha sido dictaminada únicamente en unos pocos países. Más aún, cuando estas medidas compensatorias son propuestas, ellas se focalizan en grupos de mujeres vulnerables: niñas en zonas rurales, niñas pobres y mujeres analfabetas. Confundir igual acceso con equidad significa también considerar que el conocimiento en la escuela es neutro en género; de esta manera, esta política falla en cuestionar la naturaleza de la experiencia escolarizada. ${ }^{4}$

En términos globales, ha habido mejoras en la naturaleza de la currícula escolar, pues muchas de las peores formas de estereotipos sexuales han sido eliminadas de los libros de texto. Educadores y decisores políticos han tomado más conciencia de las prácticas sexistas en los colegios, y en especial del acoso sexual. Pero, la construcción compartimentada y mutuamente excluyente de las formas de feminidad y masculinidad no solo se dan por sentadas, sino que son constantemente alimentadas.

Hoy en día, dos importantes políticas globales en educación son representadas en la Educación para Todos (EPT, adoptada unánimemente por todos los gobiernos participantes en Dakar, 2000), así como en los Objetivos de Desarrollo del Milenio (oDMs) adoptados por las Naciones Unidas (también en 2000). EFA tiene seis objetivos, dos de los cuales se refieren explícitamente al género (el objetivo $\mathrm{n}^{\circ} 2$, que busca educación primaria para todos el 2015, universalmente; y el objetivo $\mathrm{n}^{\circ} 5$, que pretende la paridad de género en los niveles de primaria y secundaria para 2005 y a todos los niveles educativos para 2015). Estos dos objetivos reflejan una vez más una preocupación exclusiva en cuanto 
Nelly P. Stromquist

al acceso e, igualmente, asumen implícitamente que el acceso, sin importar el contenido, generará el cambio en las relaciones sociales de género. Los ODMs también proponen educación primaria para mujeres y hombres universalmente y tienen un explícito objetivo relacionado con género, el $\mathrm{n}^{\circ} 3$, que hace un llamado a los países "a promover la igualdad de género y poder de la mujer". Esta meta no presenta una definición de empoderamiento; menos aún desde una perspectiva feminista. El objetivo $\mathrm{n}^{\circ} 3$ comprende cuatro indicadores: la proporción de niñas y niños en la escuela primaria y secundaria y la participación de mujeres y hombres en el ámbito universitario; la proporción de mujeres y hombres que saben leer entre 15-24 años; la cantidad de mujeres en empleos asalariados en los sectores no agrícolas y la proporción de escaños ocupados por mujeres en el congreso nacional. Estos indicadores equiparan todo tipo de educación con empoderamiento, una posición que va contra los hallazgos existentes sobre los procesos de socialización en la escolaridad formal; más aún, se centran en capacidades individuales, no en capacidades colectivas, que constituyen una caracterización crucial del empoderamiento feminista. Cabe destacar que los oDMs fueron decididos por actores gubernamentales sin la participación del movimiento de mujeres.

Los Estados son espléndidos en discursos sobre políticas y pobres en teorías de acción (ésta es la lógica que apoya intervenciones específicas); débiles en esfuerzos para la implementación; y tacaños al otorgar recursos para promover cambios en las relaciones sociales de género. La teoría feminista raramente es utilizada por los actores estatales; el interés declarado por el Estado en cuanto a género no se ha traducido en un esfuerzo teórico de entenderlo y cambiarlo. Los problemas que las mujeres enfrentan en el campo educativo son reducidos a problemas de acceso y competencia; de esta manera, consideran por deducción la escolaridad como neutra en cuanto a género. La mayoría de los gobiernos incluyen la sexualidad en la currícula de la escuela pública; pero generalmente tratan la sexualidad como un tema de salud y control, particularmente, en cuanto al VIH/SIDA.

El espacio público ocupado por el Estado, tanto en forma nacional como internacional, evita el espacio privado en varias formas: (1) la sexualidad es vista como irrelevante en la escuela, peligrosa y con riesgo de ser redefinida; (2) las propuestas que existen para ampliar la definición de la sexualidad e influir en las prácticas que limitan la autonomía de las mujeres sobre su cuerpo son ignoradas; y (3) los esfuerzos estatales 
Una cartografía social del género en educación

para mejorar el acceso a la educación fallan al considerar los aspectos ideológicos de ambos, la familia y el Estado, que limitan las opciones de las mujeres, al reproducir mentalidades de género asimétricas.

\section{Actores no estatales}

También llamada sociedad civil, estas instituciones comprenden una gama diversa. Específicamente, en cuanto a género, cuatro grupos surgen como actores claves: el movimiento de mujeres y ONGs feministas; académicos que trabajan temas de género; asociaciones de profesores; $\mathrm{y}$ grupos religiosos (en especial aquellos que representan las ideologías cristiana, islámica e hindú). Algunos de estos actores favorecen los cambios en género; otros se oponen abiertamente. Tienen distintas formas de acción en cuanto a los asuntos de género, que van desde las marchas a ejercer presión ante el gobierno para lograr nueva legislación. En el acápite siguiente, yo me apoyo primeramente en un estudio comparado de políticas públicas en educación en tres naciones latinoamericanas: Costa Rica, Perú y Brasil (Stromquist, 2006).

\section{a) Movimientos de mujeres y organizaciones feministas}

Las activistas de estos grupos promueven la acción colectiva de las mujeres. A pesar que las mujeres se encuentran fraccionadas por diferencias en raza, clase social y sexualidad, la categoría "mujer" ha probado ser una base adecuada para la movilización. Estos grupos se concentran en mujeres adultas y reclaman beneficios materiales y derechos ciudadanos. También abogan por la incorporación de género en todas los sectores de acción gubernamental, una posición tomada desde la Cuarta Conferencia Mundial de Mujeres (en Beijing, 1995) y conocida como mainstreaming. Estos grupos prestan considerable atención a la experiencia en el espacio privado de las mujeres de su vida cotidiana e intentan ampliar los derechos de las mujeres en estos ámbitos: demandando derechos sexuales y reproductivos y luchando para eliminar la violencia doméstica contra las mujeres. Una acción de importancia tomada por el movimiento feminista en el Brasil logró la expansión de la educación infantil. Generalmente, el trabajo educativo de estos grupos se centra en temas de empoderamiento y generación de ingresos. Raramente tocan la educación formal y por ello no la cuestionan. 
Los espacios físicos para las reuniones, otorgados por las ONGs y los grupos populares, constituyen importantes lugares de encuentro donde se discuten los problemas de género y se conforman las agendas. Los temas de acción incluyen ambas: políticas distributivas - aquellas que proveen nuevos beneficios simbólicos y materiales al interior de todas las categorías designadas - así como políticas estructurales o redistributivas aquellas que buscan repartir de los beneficios entre y para todas las clases, etnias, razas, géneros u otros grupos sociales.

La mayoría de los derechos que las mujeres han conseguido en las pasadas tres décadas son consecuencia del activismo de los movimientos de mujeres y de las ONGs establecidas. Con su conocimiento del espacio privado, estos grupos han superado la visión de lo político, yendo más allá de las políticas electorales y de las políticas de oficinas públicas; para incluir el poder y la carencia impotente del mismo, que existe en los niveles micro, en las relaciones íntimas y en la familia. Los grupos de mujeres, especialmente en América Latina, han contribuido grandemente a la construcción intelectual de una ciudadanía "ampliada" o "completa", lo que trae al escenario los derechos sociales, en los cuales aún se centra la concepción de ciudadanía (Sojo, 2002). El movimiento de mujeres y las organizaciones feministas buscan influir en el Estado a través de abogar y de ejercer acciones de presión; pero dado que actúan bajo dependencia económica, deben apoyarse en otros grupos más poderosos, tales como las ONGs internacionales y algunas agencias bilaterales progresistas. Estas agencias a menudo giran su atención hacia nuevos temas y, de esta manera, conceden alta vulnerabilidad a los grupos de mujeres. Generalmente, el Estado no considera a los grupos de mujeres como interlocutores importantes y trata con ellos únicamente en circunstancias excepcionales (generalmente, en respuesta a estudios y posiciones en las que las ONGS feministas cuestionan las acciones del Estado).

\section{b) Académicas feministas}

Este espacio es ocupado principalmente por mujeres y crecientemente por hombres pro-feministas, es decir, hombres que son capaces de reconocer lo positivo y de trabajar para modificar las relaciones de género.

Las académicas feministas han contribuido al cambio en el análisis de mujeres (desventaja y discriminación) a género (construcción de diferencias asimétricas, el papel de la ideología y del poder); y a la ex- 
Una cartografía social del género en educación

ploración que va más allá de las dicotomías de género. La investigación sobre educación ha registrado cambios de dirección, de centrarse al principio en la naturaleza de género de la currícula escolar en los años 70 s, a las prácticas sexistas en la escuela en los 80 s, hasta la construcción de identidades de género en los 90s; y, actualmente, al papel de los colegios en el desarrollo de una ciudadanía amplia (Dillabough, 2001; ver también Arnot et al., 1999, Arnot y Dillabough, 2000; y Moore, 2004).

En la actualidad, las académicas feministas pueden categorizarse en dos grupos: (1) Aquellas que trabajan en educación y en disciplinas como sociología, economía y ciencias políticas; que están comprometidas con temas de igualdad, equidad y con la transformación de las relaciones entre mujeres y hombres en la sociedad. Estas académicas avalan las acciones colectivas como maneras de cambiar las asimetrías en las construcciones de género. (2) Aquellas en campos de estudios tales como cultura y humanidades, que evitan categorías binarias de mujeres y hombres y que buscan desarrollar nuevas conceptualizaciones más allá de sexo y género. Estas últimas prefieren acciones individuales de resistencia; así como aproximaciones postmodernas.

El Estado guarda distancia de las académicas feministas. Eventualmente, las académicas en educación influyen al Estado mediante consultorías. Hasta donde yo conozco, las agencias estatales habitualmente no leen artículos producidos por las académicas feministas y de esta manera no se mantienen al día con los últimos debates intelectuales. Las feministas postmodernas proponen estrategias de cambio en el ámbito individual y no buscan contacto con el Estado (Cole, 2000; McRobbie, 2005).

\section{c) La religión institucionalizada}

A pesar que un gran número de países establecen en sus constituciones que existe una separación entre la religión y el poder secular, se ha encontrado que la religión organizada juega un rol de influencia no solamente en los países con religión oficial, sino también en aquellos con gobiernos laicos. La Iglesia Católica es la burocracia más grande y antigua del mundo; es igualmente una organización muy eficiente y efectiva, que mantiene cercanos contactos formales e informales con representantes del Estado oficial. En la región de América Latina, con la excepción del Bra- 
Nelly P. Stromquist

sil (un Estado laico), la Iglesia Católica ha utilizado sutiles maniobras para defender el status quo; particularmente para apoyar los valores tradicionales de la familia (esto es, la maternidad), promover un modelo único de familia, y atacar toda desviación de la heterosexualidad. La Iglesia Católica también ha tenido un papel decisivo vetando el desarrollo y uso de materiales progresistas de educación sexual.

\section{d) Asociaciones de profesores}

Las organizaciones profesionales que representan a los maestros tienden a ejercer significativa presión mediante huelgas y respaldo a determinados candidatos políticos. Sin embargo, ellos no han ido más allá en cuanto a influenciar al Estado sobre al tema de género. Su agenda incluye la defensa de escuela pública gratuita y de calidad; y, frecuentemente, están a favor de asuntos relacionados a reivindicaciones personales, tales como un presupuesto más alto para la educación pública, mejor remuneración y límites en la evaluación de rendimiento de los profesores. ${ }^{5}$

Debe observarse que dentro de la sociedad civil en el contexto latinoamericano hay grupos que defienden la educación pública con gran ahinco. Estos grupos han demostrado fuerte activismo en reuniones como el Foro Social Mundial y el Foro Mundial de Educación. La atención que estos grupos dan al género es, desgraciadamente, mínima. Uno de los más recientes documentos, La Carta de Nova Iguaçu (2006), contiene solo una breva referencia al rechazo de "toda forma de racismo y sexismo, dentro y fuera de nuestras escuelas y de nuestras universidades"

\section{El tiempo}

La cartografía social nos ha permitido explorar la vinculación de los varios campos que afectan el género en la educación. Esta aproximación es menos efectiva al incorporar la dimensión temporal. Mientras que usualmente emergen espacios múltiples al interior de un mismo contexto, algunos espacios tienden a tener su propio tiempo, así como algunas de sus instituciones se convierten en prisioneras de sus propias percepciones iniciales. En otras palabras, el tiempo afecta a todos los actores e instituciones sociales; pero, de la misma manera que las organizaciones reconocen solamente ciertas fuerzas e influencias, en algunos casos el 
Una cartografía social del género en educación

tiempo se congela. A pesar que el tiempo es difícil de mapear, pueden identificarse dos manifestaciones principales del tiempo que afectan el género y la educación.

\section{Tiempo de modernización}

Específicamente en el área de educación, la modernización pone gran énfasis en logros y estándares elevados. Medida de esta manera, la calidad de la educación es definida sin considerar el impacto de la escuela en las relaciones de género y, por ello, contribuye a debilitar todo esfuerzo para cambiar el impacto de género en la sociedad. La modernidad actualmente ha adoptado una forma de manejo de muchos aspectos de la vida social, que está basada en la vigilancia y en la desconfianza. La educación no es ya considerada como un derecho social y, consecuentemente, no se está comportando como un bien público, sino más bien como algo que puede ser obtenido privadamente. Como Ibarra-Colado (2005) observa, la educación ha sido reenmarcada como un servicio para aquellos sectores de la sociedad con capacidad de consumo.

\section{Tiempo de globalización}

El impacto de la globalización ha sido positivo y negativo para las mujeres. Por el lado positivo, muchas mujeres se han incorporado al mercado laboral y por lo tanto poseen un ingreso independiente, lo que les permite una dimensión estratégica de empoderamiento. Del otro lado, su inclusión en el mercado frecuentemente ha resultado en bajos sueldos, así como una creciente participación en el sector informal de la economía.

La expansión de una sociedad centrada en el mercado, con énfasis excesivo en el razonamiento instrumentalista, reduce el espacio para mejoras éticas y sociales. El análisis político y el activismo son considerados como innecesarios y como obstáculos para la eficiencia tecnológica, la misma que se convierte tanto en los medios como en el fin. Podríamos preguntarnos: ¿ Si la educación no se ve más como un bien común, en qué bases se puede desarrollar la solidaridad entre los miembros de la sociedad? ¿Y si la solidaridad no es asumida en los colegios, qué lugar de encuentro queda para inculcar interés por la justicia social y de género? 
Nelly P. Stromquist

\section{Progreso incierto}

El espacio público es dominado por los actores estatales, quienes prefieren decidir y planificar solos, en lugar de, en el caso de género, buscar la participación del movimiento de mujeres o feministas académicas. Hay una superposición importante entre actores estatales nacionales e internacionales, los que muestran consenso al definir temas de género en categorías fragmentadas (mujeres indigentes, niñas campesinas, familias pobres) y a través de acciones que dejan las preocupaciones ideológicas sin abordar. Las acciones del Estado se centran mayormente en la educación formal, desestimando así la mejor manera de educar para facilitar el cambio social - la educación no formal. Dentro de la educación formal, los temas de género son definidos como igualdad de oportunidades y vinculados sólo a las condiciones de los grupos de mujeres particularmente desventajados. Esta relación no es accidental, sino que más bien refleja el propósito del Estado de proteger la naturaleza patriarcal de subordinación y perenne control de las mujeres. Dado que existe un pequeño traspase de los límites por parte de los actores estatales, una limitada circulación de las teorías de género o de perspectivas feministas llega hasta el gobierno. Las fallas en señalar las inequidades de la esfera privada y de establecer enlaces con los movimientos de mujeres, activistas feministas y académicas indican que el Estado prefiere género sin feminismo así como buscar la igualdad de género sin incluir sus principales reclamos aquellos del movimiento de mujeres. Esta situación es más grave por la limitada circulación de ideas de las teorías elaboradas entre académicas feministas, y las ONGs o miembros del movimiento de mujeres. Y viceversa, actividades y productos del movimiento de mujeres y ONGs feministas son rara vez utilizados por las feministas académicas.

A pesar de los terrenos evitados como el espacio privado y los lugares explícitamente ignorados tales como las ONGs de mujeres y la universidad (donde se encuentran la mayoría de las feministas académicas), ha habido considerable progreso en la mejora de las condiciones de las mujeres y en el avance hacia una mayor igualdad de género. Entre lo logrado, podemos enumerar la presencia creciente de grupos de mujeres organizados e informados; una aceptación creciente de los derechos de las mujeres como derechos humanos fundamentales; mayor entendimiento de los factores relacionados a inequidades de género y su interconexión; un número en aumento de hombres pro-feministas - que se dan cuenta 
Una cartografía social del género en educación

de sus privilegios y que quieren modificar esto; y una mayor cantidad de dispositivos legales que aseguran los derechos políticos y económicos de las mujeres, tales como los que rigen los derechos de propiedad, divorcio, cuotas electorales, y diferentes aspectos de violencia contra las mujeres.

Haciendo un balance, ha habido mayor progreso para las mujeres en asegurar la atención y sus derechos en el espacio público que en el espacio privado. El espacio público, sin embargo, se encuentra segmentado en varios actores y puntos de vista y se caracteriza por una escasa comunicación entre los actores estatales y no estatales. Son evidentes algunos patrones: entre aquellas entidades con poder - el Estado nacional y las agencias bilaterales y multilaterales - hay una tendencia hacia posiciones convergentes; representada por un entendimiento modesto de género y limitada acción para sustentarlo. Entre aquellos sin poder político formal (las ONGs feministas y las feministas académicas) hay una creciente divergencia, con las ONGs tendiendo al reclamo por igualdad para mujeres y hombres; $y$ muchos en el mundo académico centrándose en discursos de "alcance cultural", ignorando las diferencias en las condiciones materiales y mostrándose indecisos en cuanto a asumir dicotomías de hombre y mujer.

\section{El camino hacia delante}

Cambiar nuestra comprensión de género y las relaciones sociales de género concomitantes representa una tarea difícil. En las teorías de innovación, uno de los principales principios para la adopción de ideas nuevas es su compatibilidad con los valores existentes, experiencias pasadas y necesidades de los potenciales adeptos. Las acciones de género implican justicia social y revolución cultural, de formas que cuestionan profundamente el status quo: son temas polémicos la división sexual del trabajo, los derechos sexuales y las orientaciones sexuales. Obviamente, el trabajo en género genera ideas nuevas que son incompatibles con las tradiciones e ideologías existentes.

La demanda por igualdad de género no debería ser planteada como opuesta al reconocimiento de diferencias entre mujeres. A pesar que el escenario a largo plazo lleva a una definición de sociedad en la cual la diferenciación categórica entre mujeres y hombres será reducida drásticamente; no parecería aconsejable, en el corto plazo, eliminar el uso de las categorías mujer y hombre. Tal dicotomía es esencial para delinear 
el grado de inequidad que las mujeres encaran, así como la falta de progreso alcanzado hasta aquí. Sólo desde una posición de toma de conciencia acerca de las inequidades creadas por la dicotomía de género y luego de asegurar el poder necesario para negociar las diferencias sociales podrá abandonarse esta dicotomía, para conservar una sociedad justa.

Los hombres continúan dominando el espacio público y mientras las mujeres, en la cultura popular, están hechas para dominar el espacio privado, ellas, en los hechos se encuentran relegadas a la domesticidad. Parecería que los hombres no han tenido la oportunidad de reflejarse en masculinidades alternativas. ¿Por qué? Nadie les ha impedido. ¿'Tal vez ellos no han tenido las motivaciones para explorar estos asuntos, desde su posición de privilegio? Actualmente, hay una capacitación muy reducida de las autoridades masculinas en los temas de género; y de allí, su falta de comprensión sobre el impacto de variadas intervenciones políticas sobre género (o de falta de intervenciones).

Lamentablemente, permanece insatisfecha la necesidad de vincular las teorías de género a las políticas y prácticas públicas. Existen muy pocos espacios para las mujeres en las instancias estatales. Casi no existe articulación entre el debate académico, los representantes gubernamentales y los líderes de la comunidad. Es más, la mayoría de los académicos que trabajan sobre género se encuentran bastante alejados del movimiento de mujeres. Una parte esencial del nuevo consenso debe recuperar las dimensiones materiales y prácticas y reducir el énfasis discursivo en el tema de género.

Las sociedades se encuentran constituidas por múltiples redes de poder que se sobreponen y entrecruzan en el espacio social. Esto se aplica con especial relevancia al tema de género. Ampliando la noción de espacios que se intersectan, Collins (2004) indica que las ideas en sí mismas son el producto de las redes sociales. El uso de espacio como una instancia de análisis, al discutir sobre género, no es sólo una supuesta metáfora, sino que en determinadas situaciones representa territorio y ubicación real. He buscado complicar nuestra comprensión de género en la sociedad y de género en educación demostrando la existencia y poder diferenciado de los distintos actores sociales y su ubicación. Quienes buscan una transformación de las relaciones sociales de género habitan espacios bastante distantes de aquellos que detentan el poder formal, con una brecha muy amplia entre ellos. El desarrollo de posiciones convergentes 
Una cartografía social del género en educación

entre estos actores no estatales emerge como un paso inevitable a fin de que las mujeres puedan moverse desde los márgenes hacia el centro.

Recebido em maio de 2006 e aprovado em junho de 2006.

Notas

1. Un letrado que introdujo la cartografía social para la educación comparativa es Rolland Paulston (1996).

2. La autora de esta frase es Carol Hanisch (1975) en un ensayo titulado "The personal is political”, en 1969, en la Redstockings Collection, titulado Feminist revolution y posteriormente reimpreso en 1975. Anteriormente, el sociólogo C. Wright Mills había resaltado la intersección entre temas públicos y problemas personales en su clásico The sociological imagination (1959). Aparentemente, las feministas llegaron a la misma conclusión independientemente.

3. Los Estados Unidos no han suscrito el CEDAW, así como 12 pequeños miembros de las Naciones Unidas, incluyendo la Santa Sede.

4. En los EE.uu. la paridad de género en la matrícula en el ámbito de escuela secundaria se alcanzó desde 1960. Si toda la solución radicara en el acceso a la educación, no habría existido un movimiento de mujeres en ese país en la década de los 70 .

5. El lector debe recordar que describo la situación en América Latina. En países tales como Canadá y Reino Unido, las profesoras mujeres, basadas en el hecho que ellas constituyen mayoría de miembros en las asociaciones de profesores, se han involucrado en acciones políticas hacia el cambio en género.

\section{Referencias bibliográficas}

ARNOT, M.; DAVID, M.; WEINER, G. Closing the gender gap in education. Cambridge: Polity, 1999.

ARNOT, M.; DILLABOUGH, J.-A. (Ed.). Challenging democracy: international perspectives on gender, education and citizenship. London: Routledge; Farmer, 2000.

ASHFAR, H. (Ed.). Women, State, and ideology. Albany: State University of New York, 1987.

BOWSER, B.; PATTON, J. Domestic spaces as public places: an ethnoarchaeological case study of houses, gender, and politics in the ecuadorian Amazon. Journal of Archaeological Method and Theory, New York, v. 11, n. 2, p. 157-181, 2004. 
COLE, M. (Ed.). Education, equality and human rights. London: Routledge, 2000.

COLLINS, R. Interaction ritual chains. Princeton: Princeton University, 2004.

CONNELL, R. Masculinities. St. Leonards: Allen \& Unwin, 1995.

CONNELL, R. The men and the boys. Cambridge: Polity, 2000.

DAVIES, L. The civil school and civil society: gender, democracy and development. In: Arnot, M.; Dillabough, J.-A. (Ed.). Challenging democracy: international perspectives on gender, education and citizenship. London: Routledge; Farmer, 2000. p. 278-296.

DILLABOUGH, J.-A. Gender theory and research in education: modernity traditions and emerging contemporary themes. In: FranCIS, B.; Skelton, C. (Ed.). Investigating gender: contemporary perspectives in education. Buckingham: Open University, 2001. p. 11-26.

DILLABOUGH, J.-A.; ARNOT, M. Feminist political frameworks. In: Arnot, M.; Dillabough, J.-A. (Ed.). Challenging democracy: international perspectives on gender, education and citizenship. London: Routledge; Farmer, 2000. p. 21-40.

DORE, E. The holy family. imagined households in Latin American history. In: Dore, E. (Ed.). Gender politics in Latin America: debates in theory and practice. New York: Monthly Review, 1997. p. 101-117.

FINE, M. Sexuality, education, and adolescent females: the missing discourse of desire. Harvard Educational Review, Cambridge, Mass., v. 58, n. 1, p. 29-53, 1988.

FRANCIS, B.; SKELTON, C. Introduction. In: Francis, B.; SkelTon, C. (Ed.). Investigating gender: contemporary perspectives in education. Buckingham: Open University, 2001. p. 1-7.

GROSSMAN, A. et al. Beijing betrayed; women worldwide report that governments have failed to turn the platform into action. New York: Women's Environment and Development Organization, 2005.

GUZMÁN, V. Gobernabilidad democrática y género, una articulación posible. Santiago: Unidad Mujer y Desarrollo; U.N. Economic and Social Commission, 2003. 
Una cartografía social del género en educación

HANISCH, C. The personal is political. In: REDSTOCHINGS OF THE WOMMEN'S LIBERATION MOVEMENT. Feminist revolution. New York: Random, 1975.

HARCOURT, W. Rethinking difference and equality: women and the politics of place. In: Prazniak, R.; Dirlik, A. (Ed.). Places and politics in an age of globalization. Lanham: Rowman \& Littlefield, 2001. p. 299-322.

IBARRA-COLADO, E. A Latin American response to policy changes in higher education reform: some key questions. Mexico City: Universidad Autonoma Metropolitana-Iztapalapa, 2005. (manuscrito).

KRASNER, S. Structural conflict: the Third World against global liberalism. Berkeley: University of California, 1985.

LEE, S. Sexuality and citizenship. In: Arnot, M.; Dillabough, J.-A. (Ed.). Challenging democracy: international perspectives on gender, education and citizenship. London: Routledge; Farmer, 2000. p. 259-277.

MCROBBIE, A. Feminist disorders. London: Sage, 2005.

MILLS, C.W. The sociological imagination. New York: Oxford, 1959.

MOORE, R. Education and society: issues and explanations in the sociology of education. Cambridge: Polity, 2004.

PATEMAN, C. The sexual contract. Cambridge: Polity, 1988.

PAULSTON, R. Social cartography: mapping ways of seeing social and educational change. New York: Garland, 1996.

REDCLIFT, N. Conclusion: post binary bliss: towards a new materialist synthesis? In: Dore, E. (Ed.). Gender politics in Latin America: debates in theory and practice. New York: Monthly Review, 1997. p. 222-236.

SIEKIERSKA, E. From the task force on women in cartography to the ICA working group on gender and cartography: what we learned. Ottawa: Geomatics Canada; National Resources Canada, 1995. Disponível em: <www.geo.ar.wroc.pl/GC/papers/barcelona.html>. Acesso em: 16 feb. 2005.

SOJO, C. La noción de ciudadanía en el debate latinoamericano. Revista de la CEPAL, Santiago de Chile, v. 76, p. 25-38, 2002. 
STROMQUIST, N. (Ed.). La construcción del género en las políticas públicas: perspectivas comparadas desde América Latina. Lima: Instituto de Estudios Peruanos, 2006.

STROMQUIST, N. Romancing the State: gender and power in education. Comparative Education Review, Chicago, v. 39, n. 4, p. 423-454, nov. 1995.

UNITED NATIONS DEVELOPMENT PROGRAMME. Human Development Report 2002: deepening democracy in a fragmented world. New York: UNDP, 2002. 